\title{
SEARCHING FOR NEAS FROM EARTH OR SPACE
}

\author{
A.W. HARRIS \\ Jet Propulsion Laboratory \\ Caltech, Pasadena, CA 91109 USA
}

\begin{abstract}
Different options for searching for near-Earth asteroids that may pose an impact hazard to the Earth in the future are discussed. The space option has not been studied in as much detail as the ground-based survey option, so a more detailed study of the space option might be appropriate. It is not evident, however, that a space-based system can be competitive or superior to the ground-based option, because past experience argues very strongly to the contrary.
\end{abstract}

\section{Introduction}

Impacts of asteroids or comets on the Earth pose a rare, but major threat to humanity (Chapman and Morrison, 1994). Based on this and other evaluations of the level of hazard posed by various sized events and their frequency, it appears feasible and cost effective to initiate a program to inventory all asteroids in Earth-crossing orbits, larger than $\sim 1 \mathrm{~km}$ in diameter, to determine if any one of the $\sim 1500$ such objects thought to exist might be on a collision path with the Earth in the next century. Strategies for achieving this goal have been fairly thoroughly investigated (e.g. Morrison (ed.), 1992; Harris, 1997). These studies indicate that such a survey can be successfully completed from ground-based facilities in about 10 years, at a cost of about $\$ 100 \mathrm{M}$.

In this paper, we consider the comparitive advantages of conducting such a search from space, versus from the ground. One can imagine several potential advantages of operations from space: (1) access to all wavelengths of the spectrum; (2) higher angular resolution; (3) continuous operation, that is, increased duty cycle; (4) lower sky background, i.e. improved signal-to-noise (S/N); and (5) access to more sky area. We shall discuss each of these individually below. Each of these potential advantages can be evaluated in terms of the difference in limiting magnitude of survey that could be achieved with a given system in space, compared to that on the ground. The "bottom line" is that it is practical and cost-effective to do the level of survey we have in mind at the present from the ground, and very unlikely to be faster or less expensive from space.

\section{Comparison of Earth- vs. Space-Based Surveys}

\subsection{ACCESS TO ALL WAVELENGTHS OF THE SPECTRUM}

For NEA surveys, the peak in the reflected light spectrum falls in mid-visible range, which is readily accessible from the ground. The best ground-based sites suffer less than $20 \%$ absorption at 2 air masses, and have a sky background level (mostly from air glow) only about a factor of 2 above that from space. These aspects will be addressed further in Sect. 2.4.

Thermal emission from asteroids at about $1 \mathrm{AU}$ from the Sun peaks near $10 \mu \mathrm{m}$ wavelength. This is less accessible from the ground, so space observations have a considerable advantage (cf. IRAS, ISO or SIRTF). Considering the difference in resolution of telescopes at optical vs. thermal IR, and the background sky levels at the two wavelengths, IR has a theoretical advantage of a factor of a few, but the key point is that the large element array detectors that would be needed to achieve this advantage do not exist. As will be shown below, what counts most is number of

${ }^{1}$ This paper is dedicated to the memory of Jurgen Rahe. This study was done at his request when he was Director of Solar System Exploration at NASA Headquarters. 
pixels collecting light simultaneously. Optical detectors with $\sim 10$ megapixels are readily available. IR detectors are presently limited to $\sim 100$ kilopixels (this estimate is thought to be generous), or two orders of magnitude behind optical. In addition, thermal IR detectors require liquid He cooling to achieve quantum-efficient performance. For all of these reasons, it is not believed detection by thermal emission is practical at this time, from space or from the ground.

\subsection{HIGHER ANGULAR RESOLUTION}

Detection of NEAs is noise-limited, that is, the lower the noise, the fainter the object that can be discerned. The noise level is proportional to the square-root of the background light level, which is proportional to the area of the resolution element (pixel or patch of pixels containing the image). Thus, if one can confine the image to a smaller angular area, one need only discriminate the signal above the background of a smaller patch of sky.

Let us compare a system in which the image is contained in a "box" of pixels $1^{\text {" }}$ on a side with a system of the same aperture, but containing the image within a box only $0.5^{\prime \prime}$ across. With 4 times less background area, the background level (photon count) is only $1 / 4$ as great, but the noise goes as square-root of photon count, so it is $1 / 2$ the level, thus the $0.5^{\prime \prime}$ system has twice the $\mathrm{S} / \mathrm{N}$ level of the $1^{\prime \prime}$ system. In order to recover the same $\mathrm{S} / \mathrm{N}$, the $1^{\prime \prime}$ system would have to integrate 4 times longer, since $\mathrm{S} / \mathrm{N}$ improves as the square-root of integration time. So the two systems could deliver the same threshold of detection if the $1^{\prime \prime}$ system is allowed to use 4 times longer exposures.

In practical terms, we are limited by the number of detector elements we can have. So if these two systems each have the same number of pixels in their detector (CCD) arrays, then the $1^{\prime \prime}$ system covers 4 times as much sky area in a single exposure as the $0.5^{\prime \prime}$ system. Thus taking 4 times longer exposures, it covers sky area at exactly the same rate as the $0.5^{\prime \prime}$ system, when operated to reach the same threshold detection level. This example illustrates a conclusion first reached by Greg Canavan (personal communication, 1995): as long as one avoids over-sampling the image, what counts most is the total number of pixels that can be placed in the focal plane. Applying this rule to the question of space vs. ground based surveying, the improved resolution available from space is only of value if we can place more resolution elements in the focal plane than is possible from the ground.

At present, we are limited by the size of CCD arrays, not by the number of resolution elements that can be delivered to the focal plane of a ground-based telescope. For example, presently contemplated ground-based systems are only utilizing $\sim 2^{\prime \prime}$ resolution, for the simple reason that higher resolution is not needed to completely fill the largest presently available CCD arrays. Thus the added resolution available from space is not necessary unless one were to consider a much more ambitious survey than we have dared to consider or try to justify.

\subsection{CONTINUOUS OPERATION (INCREASED DUTY CYCLE)}

System duty cycle is a very contentious question. From the ground, a telescope can only be used when the Sun is rather well below the horizon and when the Moon is below the horizon. Thus "dark" sky can only be had about $25 \%$ of the time, or $\sim 180$ hours a month. Typical observatory duty cycles, allowing for weather, equipment limitations, etc., are $\sim 15 \%$, or $\sim 100$ hours a month. In principle, one could operate $100 \%$ of the time from space, or $\sim 720$ hours a month, thus achieving a 7 -fold improvement over typical ground-based operations, or even a 4-fold improvement over the theoretical limitation of "dark sky" from the ground.

Achieving this advantage, however, is fraught with difficulties. Perhaps the duty cycle of the HST is the best model we can take as a guide. That telescope spends only $\sim 15 \%$ of its time collecting photons, the same fraction as a ground-based observatory. This is not for lack of clesire for more HST time - it is because of practical limitations that had to be faced in designing the total system. It seems unlikely that a telescope attached to the International Space Station (ISS) can do any better, considering the manned space operations that will be going on continuously in the vicinity, pointing limitations, plus all the same other constraints as faced by HST.

In the absence of an engineering study, there is little more to be said about this issue, but here especially the burden of proof lies with ISS to demonstrate that anything even as good as a $15 \%$ duty cycle can be realized. Any improvement in duty cycle over a ground-base site can be translated to an improvement in limiting magnitude of otherwise equivalent systems. The absolute maximum 
corresponding to $100 \%$ duty cycle is equivalent to an improvement of $1^{\mathrm{m}} .0$ of the threshold of detection. A factor of 4 improvement in duty cycle is equivalent to $0^{\mathrm{m}} .75$ magnitude. Equal duty cycles, as suggested by HST experience, of course corresponds to no magnitude difference. It could (likely?) be even less, leading to a negative difference for a space-based system.

\subsection{LOWER SKY BACKGROUND (IMPROVED S/N)}

The lower sky background from space translates to a higher $\mathrm{S} / \mathrm{N}$ for a given target, or to a fainter target brightness threshold for a given detection $\mathrm{S} / \mathrm{N}$. We can combine this with the modest improvement in signal due to lack of atmospheric absorption. The formulae earlier obtained (Harris, $1994,1997)$ give the threshold magnitude level obtainable from a given system in terms of a threshold $\mathrm{S} / \mathrm{N}$ that can be discriminated.

The numerical formulae have built into them background levels and atmospheric absorption appropriate for ground-based sites. If we assume that atmospheric absorption (at 2 air masses, as an average) amounts to $20 \%$, and the sky background from space is a factor of 2 lower than from the ground, that implies that the signal from space is 1.2 times greater, and the noise is $\sqrt{2}$ times less, thus the $\mathrm{S} / \mathrm{N}$ from space is improved by a factor of 1.7 . We can thus "correct" for space operation by reducing the threshold for detection by a factor of $1 / 1.7$, to obtain the appropriate limiting magnitude for a space based system. In the studies that have been done the limiting $\mathrm{S} / \mathrm{N}$ for detection was normally taken to be 4.0 . By using $\mathrm{S} / \mathrm{N}=4.0 / 1.7=2.4$, the equivalent limiting magnitude for the same system from space can be calculated. The improvement is about $0^{\mathrm{m}} \cdot 6$ magnitudes for otherwise equivalent systems.

\subsection{ACCESS TO MORE SKY AREA}

The last potential improvement from space operation is access to more sky area. It has been amply shown in several places (see, e.g., Bowell and Muinonen, 1994; Harris, 1997) that the optimum survey strategy is to cover as much sky area as possible. Ground-based observatories cannot reach down to low solar elongation, and a single observatory in either hemisphere necessarily misses a cone of sky area around the opposite hemisphere pole. In the various survey simulations that have been done, we have simulated reasonable sky access limitations for a ground-based telescope at $\sim 35^{\circ} \mathrm{N}$ latitude. More recently a simulation was done in which the horizon limitations were removed and instead a limit was set to the minimum solar elongation accessible.

A plot of the "completion curve" of such a system, using a minimum solar elongation of $45^{\circ}$, and comparing that to the one for a ground-based system at $35^{\circ} \mathrm{N}$ latitude is shown in Fig. 1. There is remarkably little difference between these curves. As described in (Harris, 1997) the relative completion curve can be scaled for systems of different capability, or to refer to objects of different size, by sliding them horizontally. As plotted, they refer to ground-based vs. space-based systems of equal magnitude threshold. By sliding the curves into rough coincidence, it is apparent that the advantage of extra sky area of the space based system is equivalent to only a difference of about 0.25 in limiting magnitude. That is, a space-based system reaching $20^{\mathrm{m}} .0$ would be equivalent to a single-site ground-based system reaching $20^{\mathrm{m}} \cdot 25$. This small difference is due almost entirely to the missing bit of sky in the south, thus siting a second ground-based telescope in the southern hemisphere should bring the two curves into almost total coincidence.

It should be noted that the simple addition of a second telescope, thereby doubling exposure times allowed, leads to an improvement of $\sim 0^{\mathrm{m}} .4$ in threshold of coverage, even more important than covering the missing hemisphere. Why does the "southern hole" account for so little? The reason is that the main area of inaccessible sky is the band surrounding the galactic plane, which cannot be used because of image confusion. That band is about $40^{\circ}$ wide $\left( \pm 20^{\circ}\right.$ galactic latitude), and is a much larger sky area than a $45^{\circ}$ cone about the southern pole, or about the Sun, for that matter.

\section{Discussion}

Of all the above effects, (1) and (2) don't matter to first order, and (3), (4), and (5) lead to improvements which can be cast in terms of improvement in the threshold limiting magnitude for otherwise identical systems, space-based vs. ground-based, as it is summarized in Table 1. 


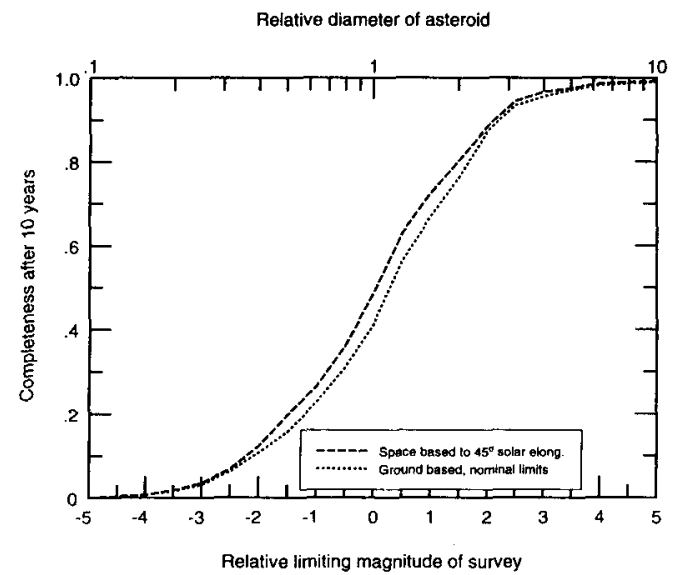

Figure 1. The relative survey completion curves for an Earth-based survey system compared to a space-based one with the same limiting magnitude capability (see text). The curves represent the fraction of NEAs detected in ten years vs. size of NEA (top scale), or equivalently, vs. system limiting magnitude for a given size object (bottom scale). As drawn, the curves could be taken to be completeness for $1 \mathrm{~km}$ objects, where the zero point of the system limiting magnitude scale is 18.5 , or equivalently, the curves could represent the completeness vs. size in $\mathrm{km}$ (top scale) for a system of limiting magnitude 18.5. It is apparent from this plot that the advantage of space operation, with respect to additional sky accessible, is equivalent to only a difference of about 0.25 in limiting magnitude of the survey.

The above potential for improvement should be considered in comparison to simply augmenting ground-based systems. As it was already noted, siting a second telescope in the opposite hemisphere essentially eliminates the $0^{\mathrm{m}} \cdot 25$ advantage due to greater sky area, so assuming that a second groundbased instrument would be placed in the opposite hemisphere, the range of potential advantage of a space-based system over a ground-based one is only $0^{\mathrm{m}} \cdot 6-1^{\mathrm{m}} \cdot 6$. How many ground-based telescopes, otherwise identical to the putative orbiting one, would be needed to achieve the same limiting magnitude? The answer is 3 to 20 , corresponding to the range $0^{\mathrm{m}} \cdot 6-1^{\mathrm{m}} \cdot 6$.

One may reasonably doubt that any improvement over ground-based duty cycle is practically feasible, so we would place the "benefit" of space operation at best equivalent to 3 ground-based telescopes of the same kind. This must be balanced against the cost of development and operation of a space-based system. Building and operating a $1 \mathrm{~m}$ telescope in orbit for 10 years seems unlikely to be less expensive than the estimated cost of $\$ 100 \mathrm{M}$ estimated for a ground-based survey. A better estimate is unavailable, but a comparison with HST, which is only twice as large a telescope, is not encouraging. A comparison even with the HST operations budget, forgetting about the cost of construction and launch, is daunting.

Turning to ground-based systems, a set of about three $1-2 \mathrm{~m}$ telescopes is about what it would take to do the Spaceguard Survey - that is, detect and catalog most NEAs larger than $\sim 1 \mathrm{~km}$ in diameter, in a decade from the start of the survey. In a report to NASA, Shoemaker et al. (1995)

TABLE 1. Improvement in the threshold limiting magnitude for otherwise identical search systems, space-based vs. ground-based

\begin{tabular}{|l|c|}
\hline Attribute & $\begin{array}{c}\text { Improvement in } \\
\text { limiting magnitude }\end{array}$ \\
\hline Duty cycle & $<0.0-1.0$ \\
Darker sky, no atm. absorption & 0.6 \\
Greater sky area & 0.25 \\
\hline Total improvement & $0.85 ?-1.85$ \\
\hline
\end{tabular}


estimated that such a system would cost $\sim \$ 100 \mathrm{M}$ to design, construct, and operate for a decade. This is about the cost of one Discovery mission, a very modest price indeed compared with other NASA programs, and minuscule compared to the ISS budget.

It is interesting to note that the cost of doing the survey is almost independent of time. At the present rate of expenditure, $\sim \$ 2 \mathrm{M} /$ year, it will take $\sim 50$ years. At a budget of $\sim \$ 10 \mathrm{M} /$ year, the time can be shrinked to $\sim 10$ years. One cannot shrink the time scale much under 10 years, because of the limits imposed by orbital dynamics: NEAs tend to be acceptably placed for discovery only about once in an orbit, of $4-5$ years period. A telescope in space could speed things up compared to the present 50-year time scale, but it could not improve much on the 10-year time scale of the $\$ 100 \mathrm{M}$ ground-based system. The reason is simply the matter of time to develop a new system, plus the intrinsic minimum time scale of $\sim 5$ years due to orbital mechanics. The earliest possible launch of an ISS telescope is 2001. Thus by the time an ISS based system could become operational, a ground-based survey could be nearing its midpoint, and "catching up" from space would be a near impossibility, regardless of how capable the system might be.

\section{Conclusion}

In the course of studying strategies for discovery surveys of near-Earth asteroids (NEAs), the possibility of doing a survey from space has been mentioned. We reported here a study of the relative effectiveness of operations from space vs. from the ground. Not surprisingly, the main factors determining effectiveness are (1) light collecting area (telescope aperture), (2) total number of resolution elements collecting light simultaneously (number of pixels for a well-scaled system), and (3) duty cycle of the system (fraction of time actually recording data). The theoretically greater sky area accessible from space makes little difference over a well-chosen ground site. Of these three factors, (1) favors ground operations over space, (2) is a technological limitation at the moment, until such time as large enough arrays of CCDs can be fabricated to capitalize on higher resolution available from space, and (3) has a theoretical advantage of a factor of a few for space operation, but has not been realized for any space-borne telescope yet ( $c f$., HST has an effective duty cycle of $\sim 15 \%$, comparable to ground-based telescopes). The good news is, it appears possible to catalog most potentially hazardous NEAs down to $\sim 1 \mathrm{~km}$ in diameter with a modest ground-based survey, over a period of about a decade. Thus there is little reason to advocate a space survey for this purpose.

\section{References}

Bowell, E, and K. Muinonen 1994, Earth-crossing asteroids and comets: Groundbased search strategies, in Hazards due to Comets and Asteroids, T. Gehrels ed., U. Arizona press, Tucson, pp. 149-197.

Chapman, C.R., and D. Morrison 1994, Impacts on the Earth by asteroids and comets: Assessing the hazard, Nature $367,33-40$.

Gehrels, T. (ed.) 1994 Hazards due to Comets and Asteroids, U. Arizona press, Tucson, 1300 pp.

Harris, A. 1994, CCD systems for searching for near-Earth asteroids, in Seventy-Five Years of Hirayama Asteroid Families, ASP Conference Series, Vol. 63, pp. 203-213.

Harris, A. 1997, Evaluation of ground-based optical surveys for near-Earth asteroids, Planetary 8 Space Science, in press.

Morrison, D. (ed.) 1995, The Spaceguard Survey. Report of the NASA International Near-Earth-Object Detection Workshop, Report prepared for NASA Office of Space Science and Applications.

Shoemaker, E.M. et al. 1995, Report of the Near-Earth Objects Survey Science Working Group, Report prepared for NASA Office of Space Science and Applications. 